Bull. Korean Math. Soc. 50 (2013), No. 4, pp. 1303-1314

http://dx.doi.org/10.4134/BKMS.2013.50.4.1303

\title{
INJECTIVELY DELTA CHOOSABLE GRAPHS
}

\author{
SEOG-Jin Kim And Won-Jin PARK
}

\begin{abstract}
An injective coloring of a graph $G$ is an assignment of colors to the vertices of $G$ so that any two vertices with a common neighbor receive distinct colors. A graph $G$ is said to be injectively $k$-choosable if any list $L(v)$ of size at least $k$ for every vertex $v$ allows an injective coloring $\phi(v)$ such that $\phi(v) \in L(v)$ for every $v \in V(G)$. The least $k$ for which $G$ is injectively $k$-choosable is the injective choosability number of $G$, denoted by $\chi_{i}^{l}(G)$. In this paper, we obtain new sufficient conditions to be $\chi_{i}^{l}(G)=\Delta(G)$. Maximum average degree, $\operatorname{mad}(G)$, is defined by $\operatorname{mad}(G)=\max \{2 e(H) / n(H): H$ is a subgraph of $G\}$. We prove that if $\operatorname{mad}(G)<\frac{8 k-3}{3 k}$, then $\chi_{i}^{l}(G)=\Delta(G)$ where $k=\Delta(G)$ and $\Delta(G) \geq 6$. In addition, when $\Delta(G)=5$ we prove that $\chi_{i}^{l}(G)=\Delta(G)$ if $\operatorname{mad}(G)<\frac{17}{7}$, and when $\Delta(G)=4$ we prove that $\chi_{i}^{l}(G)=\Delta(G)$ if $\operatorname{mad}(G)<\frac{7}{3}$. These results generalize some of previous results in $[1,4]$.
\end{abstract}

\section{Introduction}

All graphs considered in this paper are simple, finite, and undirected. We use $V(G), E(G)$ and $\Delta(G)$ to denote the vertex set, the edge set and the maximum degree of $G$, respectively. Here we introduce some notation. A $k$-vertex is a vertex of degree $k$, and a $k^{+}$-vertex is a vertex of degree at least $k$. A thread is a path with 2 -vertices in its interior and $3^{+}$-vertices as its endpoints. A $k$-thread has $k$ interior 2 -vertices, and a $k^{+}$-thread is a thread that has at least $k$ interior 2 -vertices. If $u$ and $v$ are the endpoints of a thread, then we say that $u$ and $v$ are pseudo-adjacent. For other undefined notions, we refer to [7].

An injective coloring of a graph $G$ is an assignment of colors to the vertices of $G$ so that any two vertices with a common neighbor receive distinct colors. The injective chromatic number $\chi_{i}(G)$ is the least number of colors needed for an injective coloring of $G$. Note that injective coloring is not necessarily proper, and $\chi_{i}(G)=\chi\left(G^{(2)}\right)$ where the neighboring graph $G^{(2)}$ is defined by $V\left(G^{(2)}\right)=V(G)$ and $E\left(G^{(2)}\right)=\{u v: u$ and $v$ have a common neighbor in $G\}$.

Received September 27, 2012; Revised February 18, 2013.

2010 Mathematics Subject Classification. Primary 05C15.

Key words and phrases. injective coloring, list coloring, maximum average degree, discharging.

This work was supported by the National Research Foundation of Korea (NRF) grant funded by the Ministry of Education, Science and Technology (MEST) (No. 2011-0009729). 
The girth $g(G)$ of $G$ is the length of the shortest cycle in $G$. Maximum average degree, $\operatorname{mad}(G)$, is defined by $\operatorname{mad}(G)=\max \left\{\frac{2 e(H)}{n(H)}: H\right.$ is a subgraph of $\left.G\right\}$. It follows from Euler's formula that $\operatorname{mad}(G)<\frac{2 g}{g-2}$ for every planar graph $G$ with girth at least $g$.

Given a list $L(v)$ for each vertex $v$, an injective $L$-coloring of $G$ is an injective coloring $\phi(v)$ such that $\phi(v) \in L(v)$ for every $v \in V(G)$. A graph $G$ is said to be injectively $k$-choosable if $G$ has an injective $L$-coloring for every assignment $L$ with $|L(v)| \geq k$ for every vertex $v$ in $G$. The least $k$ for which $G$ is injectively $k$-choosable is the injective choosability number of $G$, denoted by $\chi_{i}^{l}(G)$.

Note that $\Delta \leq \chi_{i}(G) \leq \chi_{i}^{l}(G) \leq \Delta^{2}-\Delta+1$ for every $G$ where $\Delta$ is the maximum degree of $G$. A natural interesting problem is to find graphs that have small injective chromatic numbers. Many researchers are interested in graphs with small injective chromatic numbers such that $\chi_{i}(G) \leq \Delta(G)+t$ for some small constant $t$.

In this direction, one of the most interesting problem is to find graphs that satisfy $\chi_{i}(G)=\Delta(G)$. For planar graphs, the following sufficient conditions in terms of $\Delta(G)$ and $g(G)$ to be $\chi_{i}(G)=\Delta(G)$ are known: $\Delta(G) \geq 71$ and $g(G) \geq 7[3]$, and $\Delta(G) \geq 4$ and $g(G) \geq 13$ [4]. Recently Borodin and Ivanova [1] studied the list version of injective coloring and proved the following results.

Theorem 1.1 ([1]). If $G$ is a planar graph of girth $g$ and maximum degree $\Delta(G)$, then $\chi_{i}^{l}(G)=\chi_{i}(G)=\Delta(G)$ in each of the following cases.

(1) $\Delta(G) \geq 16$ and $g=7$.

(2) $\Delta(G) \geq 10$ and $8 \leq g \leq 9$.

(3) $\Delta(G) \geq 6$ and $10 \leq g \leq 11$.

(4) $\Delta(G)=5$ and $g \geq 12$.

Sufficient conditions in terms of maximum average degree to have that $\chi_{i}(G) \leq \Delta(G)+t$ for some small constant $t$ were also studied. Cranston, Kim, and $\mathrm{Yu}[4]$ proved that $\chi_{i}(G)=\Delta(G)$ if $\operatorname{mad}(G) \leq \frac{9}{4}$ for $\Delta(G) \geq 4$, and $\operatorname{mad}(G) \leq \frac{42}{19}$ for $\Delta(G)=3$. Doyon, Hahn, and Raspaud [5] proved that $\chi_{i}(G) \leq \Delta(G)+3$ if $\operatorname{mad}(G)<\frac{14}{5}, \chi_{i}(G) \leq \Delta(G)+4$ if $\operatorname{mad}(G)<3$, and $\chi_{i}(G) \leq \Delta(G)+8$ if $\operatorname{mad}(G)<\frac{10}{3}$. Recently Li and $\mathrm{Xu}[6]$ studied list version of injective coloring and proved that $\chi_{i}^{l}(G) \leq \Delta(G)+4$ if $\operatorname{mad}(G)<\frac{10}{3}$ and $\Delta(G) \geq 30$, and $\chi_{i}^{l}(G) \leq \Delta(G)+2$ if $\operatorname{mad}(G)<3$ and $\Delta(G) \geq 12$.

In this paper, we obtain new sufficient conditions on $\operatorname{mad}(G)$ to be $\chi_{i}^{l}(G)=$ $\Delta(G)$, which generalizes some of the results in $[1,4]$. Our main results are as follows.

Theorem 1.2. Let $G$ be a graph with $\Delta(G) \geq 6$. If $\operatorname{mad}(G)<\frac{8 k-3}{3 k}$, then $\chi_{i}^{l}(G)=\Delta(G)$ where $\Delta(G)=k$.

In addition, when $\Delta(G)=5$ we prove that $\chi_{i}^{l}(G)=\Delta(G)$ if $\operatorname{mad}(G)<\frac{17}{7}$ in Theorem 4.2 , and when $\Delta(G)=4$ we prove that $\chi_{i}^{l}(G)=\Delta(G)$ if $\operatorname{mad}(G)<\frac{7}{3}$ in Theorem 4.1. Theorems 1.2, 4.1, and 4.2 imply the following corollary. 
Corollary 1.3. If $G$ is a planar graph with maximum degree $\Delta(G)$ and girth $g(G)$, then $\chi_{i}^{l}(G)=\chi_{i}(G)=\Delta(G)$ in each of the cases.
(a) $\Delta(G)=4$ and $g(G) \geq 14$
(b) $\Delta(G)=5$ and $g(G) \geq 12$.
(c) $\Delta(G) \geq 6$ and $g(G) \geq 10$.

Note that (b) and (c) of Corollary 1.3 are the same result as in Theorem 1.1. Hence Theorem 1.2 and Theorem 4.2 generalize (3) and (4) of Theorem 1.1. Also note that Theorem 4.1 improves the result of [4] saying that $\chi_{i}(G)=\Delta(G)$ if $\operatorname{mad}(G) \leq \frac{9}{4}$ and $\Delta(G) \geq 4$.

Remark 1.4. Let $G^{2}$ be the square of $G$ such that $V\left(G^{2}\right)=V(G)$ and $u v \in$ $E\left(G^{2}\right)$ whenever $d_{G}(u, v) \leq 2$. As one can see, injective coloring is closely related with the coloring of square of a graph (or called 2-distance coloring), which is a proper coloring and an injective coloring. The study of $\chi\left(G^{2}\right)$ has been largely focused on the well-known Wenger's Conjecture [8]. Note that $\Delta(G)+1 \leq \chi\left(G^{2}\right)$ for every graph $G$. Also, a lot of study has been done to find sufficient conditions to be $\chi\left(G^{2}\right) \leq \Delta(G)+c$ for some small constant $c$ in terms of girth $g(G)$ and $\Delta(G)$ or in terms of maximum average degree (see [2] for a good survey).

\section{Structural lemmas}

A graph $\mathrm{G}$ is injectively $\Delta$-critical if it is not injectively $\Delta$-choosable but all its proper subgraphs are injectively $\Delta$-choosable. It is easily checked that injectively $\Delta$-critical graph $G$ has no leaf or a cycle component.

We have the following simple but important property, which appeared in [1].

Remark 2.1. When $G$ is an injectively $\Delta$-critical graph, for every edge $u v$ of $G$, at least one of $u$ and $v$ has at least $\Delta$ vertices at distance 2. Otherwise, it is easy to make an injective $\Delta$-coloring of $G-u v$ into a desired coloring of $G$ by recoloring $u$ and $v$.

The following lemma is from [1].

Lemma 2.2. If $G$ is an injectively $\Delta$-critical graph, then $G$ has the following properties.

(C1) G has no $4^{+}$-thread.

(C2) Both end vertices of every 3-thread have degree $\Delta$.

(C3) At least one end vertex of every 2-thread has degree $\Delta$.

(C4) $G$ has no cycle consisting of 3-threads. That is, $G$ has no cycle $C$ such that

$C: x_{1} y_{1} y_{2} y_{3} x_{2} y_{4} y_{5} y_{6} x_{3} \cdots x_{s} y_{3 s-2} y_{3 s-1} y_{3 s} x_{s+1} \cdots x_{t} y_{3 t-2} y_{3 t-1} y_{3 t} x_{1}$, where $d\left(x_{i}\right) \geq 3$ for all $1 \leq i \leq t$ and $d\left(y_{j}\right)=2$ for all $1 \leq j \leq 3 t$.

From Remark 2.1, we have the following important lemmas. The proofs are immediately from Remark 2.1. 
Lemma 2.3. If $G$ is an injectively $\Delta$-critical graph, then $G$ has no vertex $v$ with $d(v) \leq \Delta(G)-1$ that is an endpoint of a $2^{+}$-thread such that $d\left(x_{i}\right)=2$ for $i \in\{3,4, \ldots, d(v)\}$ and $d\left(x_{1}\right)+d\left(x_{2}\right) \leq \Delta(G)+3-d(v)$ where $N_{G}(v)=$ $\left\{x_{1}, x_{2}, \ldots, x_{d(v)}\right\}$. In particular, $v$ has at least one $3^{+}$-neighbor if $v$ is an endpoint of a $2^{+}$-thread and $d(v) \leq \Delta(G)-1$.

Proof. Suppose that there is a vertex $v$ with the condition. Without loss of generality, we may assume that $x_{3}$ is on a $2^{+}$-thread. Given a list assignment $L(v)$ with $|L(v)|=\Delta(G)$ for each $v \in V(G), G^{\prime}=G-v x_{3}$ has an injective $L$ coloring by the minimality of $G$. Then we can recolor $x_{3}$ since $d(v) \leq \Delta(G)-1$. And the number of forbidden colors to $v$ is at most $\Delta(G)-1$ since $d\left(x_{1}\right)+$ $d\left(x_{2}\right)+d(v) \leq \Delta(G)+3$. Hence we can recolor $v$ and $x_{3}$ so that $G$ has an injective $L$-coloring, which contradicts the fact that $G$ is not injectively $\Delta$ choosable.

Similarly we can prove the following lemma.

Lemma 2.4. If $G$ is an injectively $\Delta$-critical graph, then $G$ has no 3-vertex $v$ that is adjacent to a vertex $x$ and two 2-vertices $y$ and $z$ such that $d(x) \leq$ $\Delta(G)-2$ and $\min \left\{d\left(y^{\prime}\right), d\left(z^{\prime}\right)\right\} \leq \Delta(G)-2$, where $y^{\prime}$ is the other neighbor of $y$ and $z^{\prime}$ is the other neighbor of $z$ other than $v$.

\section{Proof of Theorem 1.2}

The main tool used in the proofs of Theorem 1.2 is the discharging method. We assume, for contradiction, that there is a counterexample to the theorem, and we choose a counterexample to Theorem 1.2 with the fewest edges. Next, we will show that $\operatorname{mad}(G) \geq \frac{8 k-3}{3 k}$ in the discharging phase, which contradicts the assumption. This contradiction completes the proof.

From now on, let $G$ be a counterexample to Theorem 1.2 with the fewest edges. Then $G$ is an injectively $\Delta$-critical graph. Hence $G$ satisfies Lemmas $2.2,2.3$, and 2.4 .

By (C4) in Lemma 2.2, the 3-threads in $G$ form a forest, $F$. For each central vertex of each 3 -thread, we assign a sponsor as follows. Take a pendant $\Delta$ vertex $v$ in $F$. It becomes a sponsor of the central vertex of the 3 -thread $P_{v}$ where $v$ an endpoint of $P_{v}$. And then delete $P_{v}$ and repeat this assignment until $F$ is exhausted. Note that the number of pseudo-adjacent vertices in the forest $F$ is greater than the number of threads in it. Hence every central vertex of a 3 -thread can have its own sponsor.

\subsection{Discharging rules}

We define $\Delta(G)=k$ for convenient notation. We use a discharging argument with initial charge $\mu(v)=d(v)$. We will distribute the charges of vertices so that $\mu^{*}(v) \geq \frac{8 k-3}{3 k}$ for every vertex $v \in V(G)$, where $\mu^{*}(v)$ is the new charge of $v$. It implies that $\operatorname{mad}(G) \geq \frac{8 k-3}{3 k}$, which contradicts the assumption. 
In the discharging, we will use terms 'gives' and 'receives'. For example, when $\mu(x)=4$ and $\mu(y)=2$ and charge $\frac{1}{2}$ is transmitted from vertex $x$ to $y$, then the new charges are $\mu^{*}(x)=\frac{7}{2}$ and $\mu^{*}(y)=\frac{5}{2}$. In this case, we say that vertex $x$ gives charge $\frac{1}{2}$ to vertex $y$, or vertex $y$ receives charge $\frac{1}{2}$ from vertex $x$. On the other hand, if a $3^{+}$-vertex $x$ is an endpoint of a $1^{+}$-thread, then we say that $x$ is incident to a $1^{+}$-thread.

A vertex $v$ is called weak vertex if it is incident to $(d(v)-1) 2$-threads and $3 \leq d(v) \leq \Delta(G)-1$. Let $\beta=\frac{2 k-3}{3 k}$. We have the following discharging rules.

\section{Discharging Rules}

(R1) Each 2-vertex on a $2^{+}$-thread, except for the central vertex of a 3 thread, receives $\beta$ from its $3^{+}$-neighbor.

(R2) Each central vertex of a 3 -thread receives $\beta$ from its sponsor.

(R3) If $v$ is 2-vertex on a 1-thread such that $N_{G}(v)=\{x, y\}$ and both of the degrees of $x$ and $y$ are between 4 and $\Delta(G)-2$, then $v$ receives $\frac{\beta}{2}$ from each its $4^{+}$-neighbors.

(R4) If 2 -vertex $v$ on a 1 -thread is adjacent to a 3 -vertex $x$ such that (1) $x$ has exactly one 2 -vertex neighbor, or (2) $x$ has two 2 -vertex neighbors and one $(\Delta-1)^{+}$-neighbor, then $v$ receives $\frac{\beta}{2}$ from $x$.

(R5) For $\Delta \geq 7$, a 3 -vertex $v$ that is incident to exactly one 2 -thread is adjacent to a vertex $x$ such that $x$ is incident to at most $(d(x)-2)$ 2 -threads and $\frac{10 k+3}{2 k+6} \leq d(x) \leq \min \{7, \Delta(G)-2\}$, then $v$ receives $\frac{\beta}{2}$ from $x$.

(R6) If a vertex $v$ is not a weak vertex and is adjacent to a vertex $x$ with $d(x) \geq \min \{8, \Delta(G)-1\}$, then $v$ receives $\beta$ from $x$.

(R7) If $v$ is a weak vertex with $d(v)<\frac{6 k}{k+3}$ and $v$ is adjacent to vertex $x$ with $d(x) \geq 3$, then $v$ receives $2-d(v) \frac{k+3}{3 k}$ from $x$.

\subsection{Checking $\mu^{*}(v) \geq \frac{8 k-3}{3 k}$ for every vertex $v$}

We will show that $\mu^{*}(v) \geq \frac{8 k-3}{3 k}$ for each vertex $v$ after discharging. First, we will prove the following two claims.

Claim 3.1. If a vertex $v$ is not a neighbor of a weak vertex and $d(v) \geq \Delta(G)-1$, then $\mu^{*}(v) \geq \frac{8 k-3}{3 k}$ after discharging when $\Delta(G) \geq 6$.

Proof. If $d(v)=\Delta(G)$, then $v$ gives charge at most $(k+1) \beta$ to its neighbors and a vertex that is sponsored by $v$ by (R1), (R2), and (R6). Thus

$$
\mu^{*}(v) \geq k-(k+1) \beta=k-(k+1) \frac{2 k-3}{3 k}=\frac{k^{2}+k+3}{3 k} .
$$

Note that $\frac{k^{2}+k+3}{3 k} \geq \frac{8 k-3}{3 k}$ if $k \geq 6$. Hence $\mu^{*}(v) \geq \frac{8 k-3}{3 k}$. Thus

If $d(v)=\Delta(G)-1$, then $v$ gives charge at most $(k-1) \beta$ to its neighbors.

$$
\mu^{*}(v) \geq(k-1)-(k-1) \beta=(k-1)-(k-1) \frac{2 k-3}{3 k}=\frac{(k-1)(k+3)}{3 k} .
$$


Note that $\frac{(k-1)(k+3)}{3 k} \geq \frac{8 k-3}{3 k}$ if $k \geq 6$. Hence $\mu^{*}(v) \geq \frac{8 k-3}{3 k}$. This completes the proof of Claim 3.1.

Note that in (R7), $2-d(v) \frac{k+3}{3 k} \leq \beta$ only when $d(v) \geq 4$ or $d(v)=3$ and $k=6$. Hence we have that $2-d(v) \frac{k+3}{3 k} \geq \beta$ when $d(v)=3$ and $k \geq 6$. Thus if a vertex $y$ has a weak neighbor $x$, then the worst case is when all of the weak neighbors of $y$ are 3 -vertices. The proof of Claim 3.2 is based on this observation.

Claim 3.2. When $x$ is a weak vertex and $y$ is a neighbor of $x$ with $d(y) \geq 3$, then $\mu^{*}(x) \geq \frac{8 k-3}{3 k}$ and $\mu^{*}(y) \geq \frac{8 k-3}{3 k}$ after discharging.

Proof. In this case, $d(x)+d(y) \geq k+2$ by Remark 2.1. First, we will show that $\mu^{*}(x) \geq \frac{8 k-3}{3 k}$. Note that $x$ sends $(d(x)-1) \beta$ to its 2 -vertex neighbors by (R1) and receives $2-d(x) \frac{k+3}{3 k}$ from $y$ by (R7). Hence

$$
\mu^{*}(x)=d(x)-(d(x)-1) \beta+2-d(x) \frac{k+3}{3 k}=\frac{8 k-3}{3 k} .
$$

Next, we will show that $\mu^{*}(y) \geq \frac{8 k-3}{3 k}$. Suppose that $y$ is adjacent to $s$ weak vertices, denoted by $x_{1}, \ldots, x_{s}$ where $1 \leq s \leq d(y)$. Let $z$ be the vertex with the smallest degree in $\left\{x_{1}, \ldots, x_{s}\right\}$. We consider two cases.

Case (a): when $d(y) \leq \Delta(G)-1$

In this case $y$ gives at most $(d(y)-s) \beta$ to its $d(y)-s$ neighbors that are 2vertices or non-weak vertices, and gives charge to each of weak vertex neighbors $x_{1}, \ldots, x_{s}$ by $(\mathrm{R} 7)$. Hence

$$
\begin{aligned}
\mu^{*}(y) & \geq d(y)-\sum_{i=1}^{s}\left(2-d\left(x_{i}\right) \frac{k+3}{3 k}\right)-(d(y)-s) \beta \\
& \geq d(y) \frac{k+3}{3 k}-s\left(\frac{4 k+3}{3 k}-d(z) \frac{k+3}{3 k}\right)
\end{aligned}
$$

where $z$ is the vertex with the smallest degree in $\left\{x_{1}, \ldots, x_{s}\right\}$. Here note that $d(y)+d(z) \geq k+2$. We consider two subcases. If $d(z)=3$, then $d(y) \geq k-1$. Hence $s \leq d(y)=k-1$. Thus,

$$
\mu^{*}(y) \geq d(y) \frac{k+3}{3 k}-s\left(\frac{k-6}{3 k}\right) \geq \frac{(k-1)(k+3)}{3 k}-\frac{(k-1)(k-6)}{3 k} \geq \frac{8 k-3}{3 k},
$$

when $k \geq 6$. Therefore $\mu^{*}(y) \geq \frac{8 k-3}{3 k}$.

Next, when $d(z) \geq 4$, then $\frac{4 k+3}{3 k}-d(z) \frac{k+3}{3 k}$ is negative. Therefore $\mu^{*}(y)$ is minimized when $s=1$ in this case. Hence,

$$
\mu^{*}(y) \geq d(y) \frac{k+3}{3 k}-\frac{4 k+3}{3 k}+d(z) \frac{k+3}{3 k}=\frac{k+3}{3 k}(d(y)+d(z))-\frac{4 k+3}{3 k} .
$$

Therefore, when $k \geq 6, \mu^{*}(y) \geq \frac{8 k-3}{3 k}$ since $d(y)+d(z) \geq k+2$.

Case (b): when $d(y)=\Delta(G)$ 
In this case $y$ could be a sponsor of a 2-vertex on a 3 -thread. Hence we consider two subcases.

First, suppose that $y$ is a sponsor of a 2 -vertex $w$. Note that $y$ is on a 3 -thread which contains vertex $w$. Then $y$ gives at most $(k-s+1) \beta$ to its non-weak vertex neighbors and 2-vertex $w$ by (R6) and (R2), and then gives charge to each of weak vertex neighbors by (R7). Hence

$$
\begin{aligned}
\mu^{*}(y) & \geq k-\sum_{i=1}^{s}\left(2-d\left(x_{i}\right) \frac{k+3}{3 k}\right)-(k-s+1) \beta \\
& \geq k \frac{k+3}{3 k}-s\left(\frac{4 k+3}{3 k}-d(z) \frac{k+3}{3 k}\right)-\frac{2 k-3}{3 k} .
\end{aligned}
$$

Note that $\mu^{*}(y)$ is minimized when $d(z)=3$ and $s=k-1$ since $s \leq k-1$. Hence

$$
\mu^{*}(y) \geq k \frac{k+3}{3 k}-(k-1)\left(\frac{4 k+3}{3 k}-3 \frac{k+3}{3 k}\right)-\frac{2 k-3}{3 k}=\frac{8 k-3}{3 k} .
$$

Second, if $y$ is not a sponsor of a 2 -vertex, then $y$ gives at most $(k-s) \beta$ to its non-weak vertex neighbors by $(\mathrm{R} 6)$, and then gives charge to each of weak vertex neighbors by (R7). Hence

$$
\mu^{*}(y) \geq k-\sum_{i=1}^{s}\left(2-d\left(x_{i}\right) \frac{k+3}{3 k}\right)-(k-s) \beta .
$$

Note that $\mu^{*}(y)$ is minimized when $d(z)=3$ and $s=k$ since $s \leq k$. Hence

$$
\mu^{*}(y) \geq k \frac{k+3}{3 k}-k\left(\frac{4 k+3}{3 k}-3 \frac{k+3}{3 k}\right)=\frac{9 k}{3 k} \geq \frac{8 k-3}{3 k} .
$$

This completes the proof of Claim 3.2.

By Claim 3.2, we only need to consider a set of vertices that is not a weak vertex and not a neighbor of a weak vertex. From now on, we assume that $v$ is neither a weak vertex nor a neighbor of a weak vertex.

Case 1: $d(v)=2$.

If $v$ is on a $2^{+}$-thread and is adjacent to a $3^{+}$-vertex, then $v$ receives $\beta$ from its $3^{+}$-vertex neighbor. If $v$ is a central vertex of a 3 -thread, then it receives $\beta$ from its sponsor. Now consider the case when a 2 -vertex is adjacent to two $3^{+}$-vertices $x$ and $y$. If $d(x) \geq \Delta(G)-1$ or $d(y) \geq \Delta(G)-1$, then $v$ receives $\beta$ from $x$ or $y$ by (R6).

Suppose that $d(x) \leq \Delta(G)-2$ and $d(y) \leq \Delta(G)-2$. If $d(x) \geq 4, v$ receives $\frac{\beta}{2}$ from $x$ by (R3), and if $d(x)=3$ and $x$ has at most one 2-vertex neighbor, then $v$ receives $\frac{\beta}{2}$ from $x$ by $(\mathrm{R} 4)$. Similarly if $d(y) \geq 4$, or $d(y)=3$ and $x$ has at most one 2 -vertex neighbor, then $v$ receives $\frac{\beta}{2}$ from $y$. Hence the only remaining case is when $x$ or $y$ is 3 -vertex and has at least two 2 -vertex neighbors. We may assume that $d(x)=3$. Note that $x$ has exactly two 2 -vertex neighbors since if $x$ has three 2 -vertex neighbors, then $d(y) \geq \Delta(G)-1$ by Lemma 2.4. In this 
case, $x$ has a $(\Delta-1)^{+}$-neighbor by Lemma 2.4 since $d(y) \leq \Delta(G)-2$. Hence $v$ receives $\frac{\beta}{2}$ from $x$ by (R4). Similarly $v$ receives $\frac{\beta}{2}$ from $y$ by (R4) even when $d(y)=3$. Hence $\mu^{*}(v) \geq 2+2 \cdot \frac{\beta}{2}=2+\beta=\frac{8 k-3}{3 k}$.

Case 2: $d(v)=3$.

Subcase 2.1: $v$ is not incident to a 2-thread.

If $v$ has at most one 2-vertex neighbor, then $\mu^{*}(v) \geq 3-\frac{\beta}{2}=\frac{16 k+3}{6 k} \geq \frac{8 k-3}{3 k}$. Next consider the case when $v$ has exactly two 2 -vertex neighbors and one $3^{+}$-vertex neighbor $x$. If $d(x) \leq \Delta-2$, then $v$ does not send anything. If $d(x) \geq \Delta-1$, then $v$ sends $\frac{\beta}{2}$ to each of its 2 -vertex neighbors by (R4) and receives $\beta$ from $x$ by $(\mathrm{R} 6)$. Hence $\mu^{*}(v) \geq 3-2 \cdot \frac{\beta}{2}+\beta=3 \geq \frac{8 k-3}{3 k}$. Next, if $v$ is adjacent to three 2 -vertices, then $v$ does not send anything. Hence $\mu^{*}(v) \geq \frac{8 k-3}{3 k}$.

Subcase 2.2: $v$ is incident to a 2-thread.

By Lemma 2.3, $v$ is incident to at most two 2-threads. If $v$ is incident to exactly two 2 -threads and is adjacent to a $3^{+}$-vertex $x$, then $v$ is a weak vertex and $\mu^{*}(v) \geq \frac{8 k-3}{3 k}$ by Claim 3.2 .

Now suppose that $v$ is incident to exactly one 2-thread. Let $x$ and $y$ be the two neighbors of $v$ that are not on the 2-thread. Then $d(x)+d(y) \geq \Delta+1$ by Lemma 2.3.

In this case, we have two subcases; one case is when $\max \{d(x), d(y)\} \geq$ $\Delta(G)-1$, and the other case is when $\max \{d(x), d(y)\} \leq \Delta(G)-2$.

First, consider the case when $\max \{d(x), d(y)\} \geq \Delta(G)-1$. We may assume that $d(x) \geq \Delta(G)-1$. In this case, $v$ sends $\beta$ the 2 -vertex neighbor on the 2 -thread by (R1) and sends $\frac{\beta}{2}$ to $y$ when $d(y)=2$ by (R4), and $v$ receives $\beta$ from $x$ by $(\mathrm{R} 6)$. Hence $\mu^{*}(v) \geq 3-\frac{3 \beta}{2}+\beta=3-\frac{\beta}{2} \geq \frac{8 k-3}{3 k}$.

Next, consider the case when $\max \{d(x), d(y)\} \leq \Delta(G)-2$. Then neither $x$ nor $y$ is 2 -vertex since $d(x)+d(y) \geq \Delta+1$ and $\max \{d(x), d(y)\} \leq \Delta(G)-$ 2. Also $x$ is incident to at most $(d(x)-2)$ 2-threads, and $y$ is incident to at most $(d(y)-2) 2$-threads by Lemma 2.3 . When $\Delta \geq 8$, we have that $\frac{10 k+3}{2 k+6} \leq 5 \leq \max \{d(x), d(y)\} \leq \Delta-2$. Also when $\Delta=7$, we have that $\frac{10 k+3}{2 k+6}=\frac{73}{20} \leq 4 \leq \max \{d(x), d(y)\} \leq \Delta-2$. If $\max \{d(x), d(y)\} \geq 8$, then $v$ receives $\beta$ from $x$ or $y$ by $(\mathrm{R} 6)$. If $\max \{d(x), d(y)\} \leq 7$, then $v$ receives $\frac{\beta}{2}$ from $x$ or $y$ by (R5). Hence $\mu^{*}(v) \geq 3-\beta+\frac{\beta}{2} \geq \frac{8 k-3}{3 k}$ when $\Delta \geq 7$. When $\Delta=6, v$ sends $\beta$ its 2 -vertex neighbor and does not receives anything. Hence $\mu^{*}(v)=3-\beta=3-\frac{1}{2}=\frac{5}{2}=\frac{8 k-3}{3 k}$.

Case 3: $d(v)=4$.

If $v$ is not incident to a 2 -thread, then $v$ sends at most $d(v) \frac{\beta}{2}$ to its neighbors by (R3) since $v$ is not a neighbor of a weak vertex. Then $\mu^{*}(v) \geq d(v)-d(v) \frac{\beta}{2}=$ $d(v) \frac{4 k+3}{6 k} \geq \frac{8 k-3}{3 k}$. 
Now consider the case when $v$ is incident to a 2-thread. Note that $v$ is incident to at most three 2-threads. If $v$ is incident three 2-threads, then $v$ is a weak vertex and $\mu^{*}(v) \geq \frac{8 k-3}{3 k}$ by Claim 3.2 .

Next, consider the case when $v$ is adjacent to exactly two 2-threads. Let $x$ and $y$ be the two neighbors of $v$ that are not on the two 2-threads. Then $d(x)+d(y) \geq \Delta$ by Lemma 2.3. Without loss of generality, we may assume that $d(x) \leq d(y)$. First, when $d(y) \geq d(x) \geq 4, v$ sends $\beta$ to each of 2 -vertex neighbors on the 2 -threads. Hence $\mu^{*}(v) \geq 4-2 \cdot \beta \geq \frac{8 k-3}{3 k}$.

Second, when $d(x) \leq 3$, we have that $d(y) \geq k-3$. Note that $v$ may give charge $\frac{\beta}{2}$ to $x$ by (R3) or (R5). When $k \geq 11, v$ receives $\beta$ from $y$ by (R6) since $d(y) \geq 8$. Thus $\mu^{*}(v) \geq 4-2 \cdot \beta-\frac{\beta}{2}+\beta \geq \frac{8 k-3}{3 k}$. Next, when $7 \leq k \leq 10$, we have that $d(y) \geq 4$. Hence $v$ does not send any charge to $y$. Thus we have that $\mu^{*}(v) \geq 4-2 \cdot \beta-\frac{\beta}{2}=\frac{14 k+15}{6 k} \geq \frac{8 k-3}{3 k}$. Next, when $k=6$, we have $d(y) \geq 3$. Thus $v$ does not give any charge to $y$, since $v$ does not give any charge to $3^{+}$-vertex neighbor when $k=6$. Thus $\mu^{*}(v) \geq 4-2 \cdot \beta-\frac{\beta}{2} \geq \frac{8 k-3}{3 k}$.

Next, consider the case when $v$ is incident exactly one 2-thread. Let $N(v)=$ $\left\{x_{1}, x_{2}, x_{3}, x_{4}\right\}$ with $d\left(x_{1}\right) \leq d\left(x_{2}\right) \leq d\left(x_{3}\right) \leq d\left(x_{4}\right)$. Clearly $x_{1}$ is 2-vertex on the 2-thread. Note that $d\left(x_{2}\right)+d\left(x_{3}\right)+d\left(x_{4}\right) \geq \Delta(G)+2$ by Remark 2.1. Hence when $k \geq 8, d\left(x_{4}\right) \geq 4$. Thus $\mu^{*}(v) \geq 4-\beta-2 \cdot \frac{\beta}{2} \geq \frac{8 k-3}{3 k}$. When $k=7, v$ may send $\frac{\beta}{2}$ to each of $x_{2}, x_{3}$, and $x_{4}$ when $d\left(x_{4}\right)=3$. But, $\mu^{*}(v) \geq 4-\beta-3 \frac{\beta}{2}=\frac{14 k+15}{6 k} \geq \frac{8 k-3}{3 k}$. When $k=6$, note that $d\left(x_{4}\right) \geq 3$, so $v$ does not send charge to $x_{4}$. Hence $\mu^{*}(v) \geq 4-\beta-2 \cdot \frac{\beta}{2}=4-2 \beta \geq \frac{8 k-3}{3 k}$.

Therefore $\mu^{*}(v) \geq \frac{8 k-3}{3 k}$ for each vertex $d(v)=4$.

Case 4: $5 \leq d(v) \leq 7$ and $d(v) \leq \Delta-2$.

If $v$ is not incident to a 2 -thread, then $\mu^{*}(v) \geq d(v)-d(v) \frac{\beta}{2}=d(v) \frac{4 k+3}{6 k} \geq$ $\frac{8 k-3}{3 k}$ since (R6) does not apply to.

Consider the case when $v$ is incident to a 2 -thread. Note that $v$ is incident to at most $(d(v)-1) 2$-threads. If $v$ is incident to $(d(v)-1) 2$-threads, then $v$ is a weak vertex and $\mu^{*}(v) \geq \frac{8 k-3}{3 k}$ by Claim 3.2 .

Now suppose that $v$ is incident to $m 2$-threads where $m \leq d(v)-2$. Hence $v$ sends $m \beta$ to the neighbors on incident 2 -threads and sends at most $\frac{\beta}{2}$ to each of the other neighbors, since $v$ is not a weak vertex and not a neighbor of a weak vertex. Thus

$$
\mu^{*}(v) \geq d(v)-(d(v)-2) \beta-2 \cdot \frac{\beta}{2}=d(v) \frac{k+3}{3 k}+\frac{2 k-3}{3 k}
$$

Note that $d(v) \frac{k+3}{3 k}+\frac{2 k-3}{3 k} \geq \frac{8 k-3}{3 k}$ if $d(v) \geq \frac{6 k}{k+3}$. Thus $\mu^{*}(v) \geq \frac{8 k-3}{3 k}$ when $d(v) \geq 6$. When $d(v)=5, v$ sends $4 \beta$ to its neighbors only when $v$ is incident to exactly three 2 -threads and is adjacent to two 3 -vertices. This case happens only when $k \leq 7$ by Lemma 2.3. Thus $k=7$ since $d(v) \leq \Delta-2$. In this case, $d(v) \geq \frac{42}{10}=\frac{\overline{6} k}{k+3}$. Hence $\mu^{*}(v) \geq \frac{8 k-3}{3 k}$ for all $v$ with $5 \leq d(v) \leq 7$.

Case 5: $d(v) \geq \min \{8, \Delta-1\}$. 
First, if $d(v) \geq \Delta-1$, then $\mu^{*}(v) \geq \frac{8 k-3}{3 k}$ by Claim 3.1. If $8 \leq d(v) \leq \Delta-2$, then $v$ sends at most $d(v) \beta$ to its neighbors since it is not a neighbor of a weak vertex. Hence

$$
\mu^{*}(v) \geq d(v)-d(v) \beta=d(v) \frac{k+3}{3 k} \geq \frac{8 k-3}{3 k},
$$

since $d(v) \geq 8$. Hence $\mu^{*}(v) \geq \frac{8 k-3}{3 k}$.

Therefore we show that $\mu^{*}(v) \geq \frac{8 k-3}{3 k}$ for every vertex $v$ in $G$. This completes the proof of Theorem 1.2.

\section{Maximum average degree condition when $\Delta(G)=4$ or 5}

Theorem 4.1. Let $G$ be a graph with $\Delta(G)=4$. If $\operatorname{mad}(G)<\frac{7}{3}$, then $\chi_{i}(G)=$ 4.

Proof. Let $G$ be a minimal counterexample. The reducible configurations in Lemma 2.2, Lemma 2.3, and Lemma 2.4 also can apply to $G$. We use a discharging argument with initial charge $\mu(v)=d(v)$. We will show that $\mu^{*}(v) \geq \frac{7}{3}$ for every vertex $v \in V(G)$, which contradicts the assumption. We have the following discharging rules.

(R1) Each 2-vertex on a $2^{+}$-thread, except for the central vertex of a 3 thread, receives $\frac{1}{3}$ from its $3^{+}$-neighbor.

(R2) Each central vertex of a 3 -thread receives $\frac{1}{3}$ from its sponsor.

(R3) If $v$ is 2 -vertex on a 1 -thread, then $v$ receives $\frac{1}{6}$ from each of its $3^{+}$neighbors.

Now we will show that $\mu^{*}(v) \geq \frac{7}{3}$ for all $v \in V(G)$.

If $d(v)=2$, then it receives $\frac{1}{3}$ from its $3^{+}$-neighbor or its sponsor. Hence $\mu^{*}(v)=2+\frac{1}{3}=\frac{7}{3}$. Now suppose that $d(v)=3$. If $v$ is not incident to a 2 thread, then $v$ sends at most $\frac{1}{6}$ to each of its neighbors. Hence $\mu^{*}(v) \geq 3-3 \cdot \frac{1}{6} \geq$ $\frac{7}{3}$. If $v$ is incident to a 2-thread, then $v$ has at least one $3^{+}$-vertex neighbor. Hence $v$ sends charge to at most two neighbors. Hence $\mu^{*}(v) \geq 3-2 \cdot \frac{1}{3}=\frac{7}{3}$. If $d(v)=4$, then $v$ may send total $5 \cdot \frac{1}{3}$ to its neighbors and its sponsor. Hence $\mu^{*}(v) \geq 4-5 \cdot \frac{1}{3}=\frac{7}{3}$. Therefore $\mu^{*}(v) \geq \frac{7}{3}$ for all $v \in V(G)$.

Theorem 4.2. Let $G$ be a graph with $\Delta(G)=5$. If $\operatorname{mad}(G)<\frac{17}{7}$, then $\chi_{i}(G)=5$.

Proof. Let $G$ be a minimal counterexample. The reducible configurations in Lemma 2.2, Lemma 2.3, and Lemma 2.4 also can apply to $G$. We use a discharging argument with initial charge $\mu(v)=d(v)$. We will show that $\mu^{*}(v) \geq \frac{17}{7}$ for every vertex $v \in V(G)$, which contradicts the assumption. We have the following discharging rules.

(R1) Each 2-vertex on a $2^{+}$-thread, except for the central vertex of a 3 thread, receives $\frac{3}{7}$ from its $3^{+}$-neighbor. 
(R2) Each central vertex of a 3 -thread receives $\frac{3}{7}$ from its sponsor.

(R3) If $v$ is 2 -vertex on a 1 -thread, then $v$ receives $\frac{3}{14}$ from each of its $3^{+}$neighbors.

(R4) If 3-vertex $v$ is incident to two 2 -threads and has a $4^{+}$-vertex neighbor $x$, then $v$ receives $\frac{2}{7}$ from $x$.

(R5) If 3-vertex $v$ is incident to three 1-threads and is pseudo-adjacent to a $4^{+}$-vertex $x$, then $v$ receives $\frac{1}{14}$ from $x$.

Now we will show that $\mu^{*}(v) \geq \frac{17}{7}$ for all $v \in V(G)$.

Case 1: $d(v)=2$.

If $v$ is on a $2^{+}$-thread, then $v$ receives $\frac{3}{7}$ from its $3^{+}$-neighbor or its sponsor. If $v$ is on a 1-thread, then $v$ receives $\frac{3}{14}$ from each its $3^{+}$-neighbors. Hence $\mu^{*}(v)=\frac{17}{7}$.

Case 2: $d(v)=3$.

If $v$ is not incident to a 2-thread, then $v$ sends at most $\frac{3}{14}$ to each of its neighbors. On the other hand, each vertex $x$ that is pseudo-adjacent to $v$ has degree at least 4 . Thus $v$ receives $\frac{1}{14}$ from $x$ by $(\mathrm{R} 5)$. Hence $\mu^{*}(v) \geq$ $3-3 \cdot \frac{3}{14}+\frac{1}{14}=\frac{17}{7}$. Now suppose that $v$ is incident to a 2 -thread. Then $v$ is incident to at most two 2-threads. If $v$ is incident to a 2-thread and has two $3^{+}$-vertex neighbors, then $v$ sends $\frac{3}{7}$ to the 2 -vertex neighbor on the 2 -thread. Then $\mu^{*}(v) \geq 3-\frac{3}{7} \geq \frac{17}{7}$. If $v$ is incident to a 2 -thread and has exactly one $3^{+}$-vertex neighbor $x$, then $d(x) \geq 4$ by Lemma 2.4. Then $v$ receives $\frac{2}{7}$ from $x$ by $(\mathrm{R} 4)$. Hence $\mu^{*}(v) \geq 3-2 \cdot \frac{3}{7}+\frac{2}{7}=\frac{17}{7}$. Note that $v$ is not incident to three 2-threads.

Case 3: $d(v)=4$.

Note that $v$ is not incident to four 2 -threads. Hence the charge that $v$ sends to its neighbors is maximized when $v$ is incident three 2-threads and is adjacent to a 3 -vertex that is incident to a 2 -thread. In this case, $\mu^{*}(v) \geq 4-3 \cdot \frac{3}{7}-\frac{2}{7}=$ $\frac{17}{7}$.

Case 4: $d(v)=5$.

The worst case is when $v$ sends charge $6 \cdot \frac{3}{7}$ to its neighbors and its sponsor. Then $\mu^{*}(v) \geq 5-6 \frac{3}{7}=\frac{17}{7}$.

Therefore $\mu^{*}(v) \geq \frac{17}{7}$ for all $v \in V(G)$.

Remark 4.3. Let $G$ be a planar graph consisting of two $k$-vertices $x$ and $y$ and $2 k 2$-vertices such that $x$ and $y$ are connected by vertex-disjoint $k 2$-threads. Then $\chi_{i}(G)=k+1=\Delta(G)+1$ and $\operatorname{mad}(G)=\frac{3 k}{k+1}$. It is an interesting problem to find the sharp bound for maximum average degree to guarantee that $\chi_{i}(G)=\Delta(G)$. We have the following question.

Question 4.4. Is it true that $\chi_{i}(G)=\Delta(G)$ whenever $\operatorname{mad}(G)<\frac{3 \Delta(G)}{\Delta(G)+1}$ ? 


\section{References}

[1] O. V. Borodin and A. O. Ivanova, List injective colorings of planar graphs, Discrete Math. 311 (2011), no. 2-3, 154-165.

[2] - 2-distance $(\Delta+2)$-coloring of planar graphs with girth six and $\Delta \geq 18$, Discrete Math. 309 (2009), no. 23-24, 6496-6502.

[3] Y. Bu, D. Chen, A. Raspaud, and W. Wang, Injective coloring of planar graphs, Discrete Appl. Math. 157 (2009), no. 4, 663-672.

[4] D. W. Cranston, S.-J. Kim, and G. Yu, Injective colorings of sparse graphs, Discrete Math. 310 (2010), no. 21, 2965-2973.

[5] A. Doyon, G. Hahn, and A. Raspaud, Some bounds on the injective chromatic number of graphs, Discrete Math. 310 (2010), 585-590.

[6] R. Li and B. Xu, Injective choosability of planar graphs of girth five and six, Discrete Math. 312 (2012), no. 6, 1260-1265.

[7] D. B. West, Introduction to Graph Theory, Prentice Hall Inc., Upper Saddle Rive, NJ, 2001.

[8] G. Wenger, Graphs with given diameter and a coloring problem, Technical Report, University of Dortmund, 1977.

SEOG-JIN KIM

Department of Mathematics Education

KONKUK UNIVERSITY

Seoul, 143-701, Korea

E-mail address: skim12@konkuk.ac.kr

WON-JIN PARK

Department of Mathematics

Seoul National University

SEOUl, 151-742, KoreA

E-mail address: wonjin96@snu.ac.kr 